\title{
Effect of polarity reversal on third harmonics of cosmic ray intensity on quiet days
}

\author{
M. K. Richharia \\ Department of Physics, Govt. Science College (Autonomous) Jabalpur (M.P.) India
}

Email address:

mkrichharia@yahoo.com

To cite this article:

M. K. Richharia. Effect of Polarity Reversal on Third Harmonics of Cosmic Ray Intensity on Quiet Days. International Journal of Astrophysics and Space Science. Special Issue: Modulation of Cosmic Rays, Inter Planetary Magnetic Field, Magnetic Storm, Large and Small Amplitude Wave Train. Vol. 3, No. 2-1, 2015, pp. 1-3. doi: 10.11648/j.ijass.s.2015030201.11

\begin{abstract}
The cosmic ray (CR) intensity data recorded with Goose Bay Neutron Monitoring Station have been investigated on 60 quietest days (QD) in a year for studying the variation in tri diurnal anisotropy during solar cycle 21 and 22. It has been observed that in spite of abrupt change in the amplitude and phase of tri-diurnal anisotropy in CR intensity, the amplitude is quite significant throughout period of investigation with small amplitude during 1981 and 1990. Further, the phase shift to earlier hours during 1990-91 showing the dependence on the polarity of solar magnetic field, which is attributed to drift effect. Thus, the tri-diurnal anisotropy clearly shows 11 year type variation at mid-latitude neutron monitoring station.
\end{abstract}

Keywords: Cosmic Rays/ Higher Harmonics/Geo-magnetically Quiet Days

\section{Introduction}

The spatial anisotropy of the galactic cosmic ray intensity in the interplanetary space manifests itself as daily variation with a period of 24 hours (and its higher harmonics) due to the rotation of the Earth in the course of a day. The power Spectrum analysis as well as the Fourier analysis of the long term data of the 24-hour values of cosmic ray (CR) intensity observed by Earth based detectors have provided daily variation of extraterrestrial origin [Fujii et al 1971; Ahluwalia and Singh 1973a]. However, the amplitude of the fourth harmonics is still controversial [Ahluwalia and Singh 1973b; Pomerantz and Duggal 1971; Rao 1972; Venkatesan and Badruddin 1990; Agrawal 1981]. Moreover, it has been observed that the amplitude and phase of tri diurnal anisotropy of CR intensity on quiet days vary considerably from one period to another. On the long term behaviour of the first three harmonics showed that high degree of year to year variability exists, a trend with solar activity was evident. The studies of the higher harmonics in the daily variation of cosmic ray provided valuable information as to the nature of the cosmic ray modulation in the heliosphere.

\section{Analysis of the Data}

Solar daily variation has been studied in terms of helio- magnetic activity. A new concept of data analysis has been introduced for studying the long/short term daily variation in CR intensity recorded with neutron monitors. Fourier technique has been applied on different types of group of days chosen according to their different geomagnetic condition.

All days: This means all the $365 / 366$ days in year. Thus, these days are termed as AD. of course ignoring the days with abrupt changes.

Quiet days: Those days on which the transient magnetic variation are regular and smooth are said to be magnetically quiet or $\mathrm{Q}$ days. The criteria is based upon Ap and Kp values. There are two types of days.

60 Quiet days: According to solar geophysical data (SGD) lowest mean order number are the five quietest days in a month. Thus, $60 \mathrm{Q}$ days in a year; termed as $60 \mathrm{QD}$.

120 Quiet days: First ten quiet days in a month. Thus, 120 Q Days in a year; termed as 120 Quiet days.

The pressure corrected hourly CR intensity data (corrected for meteorological effects) on geomagnetically five quietest days (QD) in every month for Goose Bay (Lat: $53.33^{0} \mathrm{~N}$; Cutoff rigidiy; $0.52 \mathrm{GV}$; Longitude; $299.58^{0} \mathrm{E}$; Altitude; $46 \mathrm{~m}$ ) neutron monitoring station and for the period 1978-94, have been used in Fourier analysis. After applying the trend 
corrections, such a set of data have been subjected to Harmonic analysis for each day [Kumar et al 2002]. The average values of the amplitude and phase (local time of the station) of the third (tri diurnal) harmonics on yearly basis have been obtained. According to solar geophysical data five quietest days are selected in a month; thus 60 quietest days are obtained in a year. These days are called international quiet days (QD). The days with extra ordinary large amplitude if any, have not been considered. Further, the variation in the tri-diurnal anisotropy with the reversal of polarity of solar magnetic field (PSMF) on 60 QD has been also investigated. Also all those days are discarded having more than three continuous hourly data missing.

\section{Results and Discussion}

The yearly average amplitude (\%) and phase (Hrs) of tridiurnal anisotropy of cosmic ray intensity along with average value of Ap indices on Quiet Days, solar wind velocity and PSMF in the northern and southern hemisphere of the Sun for Goose Bay Neutron Monitoring Station have been plotted in Fig. 1 during the period 1978-94. It is apparent from Fig.1 that there is no systematic variation is observed in the amplitude and phase of the third harmonics of daily variation. However, some of the significant observations are such as the amplitude is decreased during the year 1981 and 1990, which coincides with phase reversal of the solar poloidal field [Kumar et al 1998]. It is observed that the amplitude is statistically nearly constant high value during the year 1986-89. The amplitude of the tri-diurnal anisotropy of CR intensity has been observed almost equal during the two consecutive solar maximum activity periods. i.e., (year 1979 and 1991) of solar cycles-21 and 22 supporting 11- year variation in the tri-diurnal anistotropy of CR intensity on quiet days [Richharia et al 1999]. Further, the amplitude of third harmonics during the years, 1982 and 1994 (Difference of 11 year) having the same value, which is again confirm the 11 year type variation in third harmonics of daily variation. The error $=0.005 \%$ contained in the amplitudes of third harmonics on quiet days. Furthermore, it is also apparent form Figure 1 that the phase has been observed during the year 1987-90 into later hours. However, a significant change towards earlier hours is observed in the phase of tri-diurnal anisotropy, when the polarity of solar magnetic field is reversed during the period 1990-91 [El Borie et al 1995a]. The phase of tri-diurnal anisotropy is shifted towards early hours during the succeeding years 1991-92, when polarity of solar magnetic field of $\mathrm{NH}$ is positive. Thus, the change in the phase of tri-diurnal anisotropy of CR intensity has been found quite significant. It is clear that there exist a 11 year type of variation in third harmonics of $\mathrm{CR}$ intensity on QD due to the variation of solar activity [El Borie et al 1995b]. The polarity dependence of the phase shift change has been interpreted as a result due to the change of CR density distribution in space caused by the difference of CR drift motion in the positive and negative polarity state [Munakata et al 1986; Nagashima et al 1986; Kumar et al 2002]. The existence of polarity dependence in the tri-diurnal variation may be defined as the state is positive, when the magnetic field is away from the sun at the north pole and toward the Sun at the south pole, while it is called negative, when the polar magnetic field are reversed [Nagashima et al 1989; Richharia et al 2007].

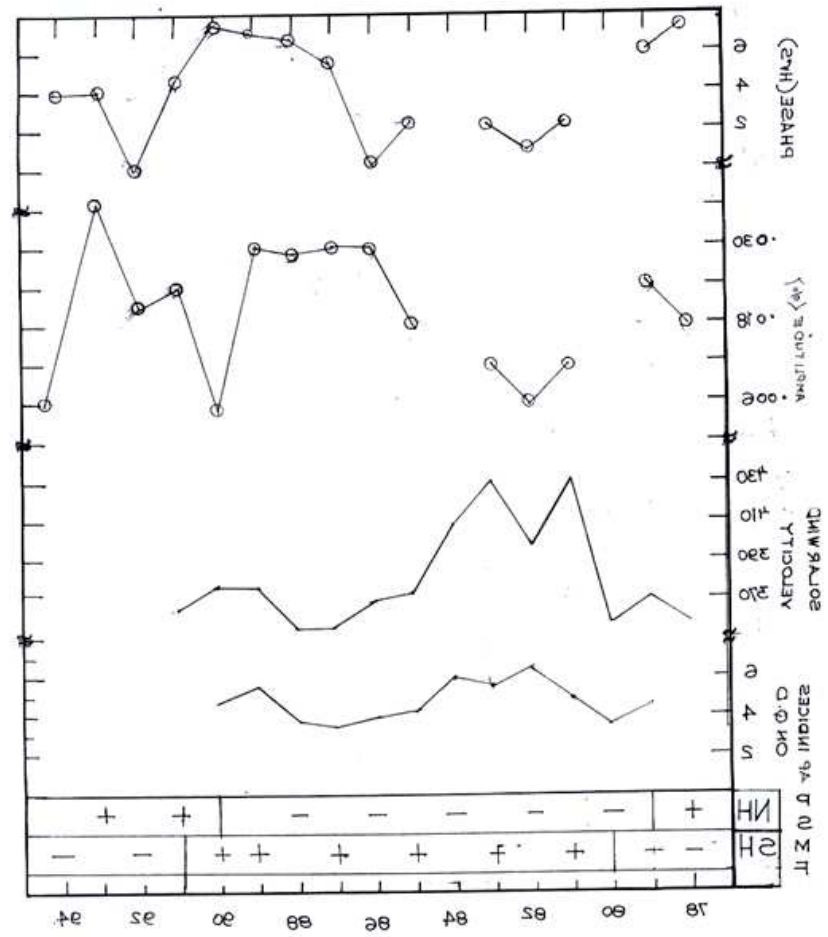

Fig.1. Amplitude(\%)and Phase (hrs) of third harmonics along with average values of Appendices on Quiet days, solar wind velocity and PSMF in the Northern and Southern hemisphere of the Sun at Bay Neutron Monitoring Station during 1978-84. 


\section{Conclusions}

The following conclusions may be drawn from the present investigation of cosmic ray intensity recorded in Goose Bay Neutron Monitoring station.

1. The amplitude of third harmonics of daily variation on QD during 1981 and 1990 is significantly low, which coincides with the phase reversal of solar poloidal magnetic field.

2. The tri diurnal anisotropy of CR intensity on $\mathrm{QD}$ has shown long term variation i.e., 11 years type variation.

3. The tri diurnal phase is shifted to later hours during the negative polarity of PSMF in NH; whereas it is shifted to early hours during positive polarity of PSMF in NH. Since the phase shift of tri-diuranl anisotropy towards early/later hours has been attributed to the change in the polarity of the PSMF, it may be derived from here that this phase shift is due to drift effect.

\section{Acknowledgments}

The authors are indebted to various experimental groups; in particular, Profs. M. Bercovitch, K. Nagashima and Miss Aoi Inoue for providing neutron monitor data.

\section{References}

[1] Agrawal, S. P. 1981, Journal Geophys. Res., 86: 10115.

[2] Ahluwalia, H. S. and Singh, S. 1973a, Proc. $13^{\text {th }}$ Int. Cosmic Ray Conf., Australia, 2:948.
[3] Ahluwalia, H. S. and Singh, S. 1973b, Proc. $13^{\text {th }}$ Int. Cosmic Ray Conf., Australia, 5:3129.

[4] El Borie, M. A., Sabbah, I.S., Darwish, A. A. and Bishra, A. A. 1995a, $24^{\text {th }}$ Int. Cosmic Ray Conf., Rome, Italy, 4:619.

[5] El Borie, M. A., Sabbah, I.S., Darwish, A. A. and Bishra, A. A. 1995b, $24^{\text {th }}$ Int. Cosmic Ray Conf., Rome, Italy, 4:603.

[6] Fujii, A., Nagashima, K., Fujimoto, K., Ueno, H. And Kondo, I. 1971, 12th ICRC, Hobart Tasmania, 2, 666.

[7] K. Munakata and K. Nagashima. 1986, Planet Space Sci. 34, 99.

[8] K. Nagashima, R. Tatsuoka and K. Munakata. 1986, Planet Spcae Sci. 34, 469.

[9] K. Nagashima and K. Fujimoto. 1989, Planet Space Sci. 37, 1421.

[10] Kumar, S., Shrivastava, S. K., Dubey, S. K., Richharia, M. K. And Gulati, U. 1998, Ind. J. Radio and Space Phys., 27, 236.

[11] Kumar, S., Agrawal, R., Mishra, R. And Dubey, S. K. 2002, Bull Astronomical Soc. India, 30, 451.

[12] Kumar, S., Agrawal, R., Mishra, R., Dubey, S. K. And Richharia, M. K. 2002, Indian J. Phy. 76 B (3), 259.

[13] Pomerantz, M. A. and Duggal, S. P. 1971, Space Sci. Rev., 12, 75.

[14] Rao, U. R. 1972, Space Sci. Rev., 12, 719.

[15] Richharia, M. K., Shrivastava, S. K. and Kumar, S. 1999, J. Pure \& Applied Physics 11, 1, p-11.

[16] Richharia, M.K. and Shrivastava, S.K. 2007, $30^{\text {th }}$ Int. Cosmic Ray Conf., Merida, Mexico, 3.2, 94.

[17] Venkatesan, D. and Badruddin. 1990, Space Sci. Rev., 52, 121. 